Journal of Behavioral Data Science, 2020, 1 (2), 156-177.

DOI: https://doi.org/10.35566/jbds/v1n2/p7

\title{
Structural Equation Modeling using Stata
}

\author{
Meghan K. Cain ${ }^{1[0000-0003-4790-4843]}$ \\ StataCorp LLC, College Station, TX 77845, USA \\ mcain@stata.com
}

\begin{abstract}
In this tutorial, you will learn how to fit structural equation models (SEM) using Stata software. SEMs can be fit in Stata using the sem command for standard linear SEMs, the gsem command for generalized linear SEMs, or by drawing their path diagrams in the SEM Builder. After a brief introduction to Stata, the sem command will be demonstrated through a confirmatory factor analysis model, mediation model, group analysis, and a growth curve model, and the gsem command will be demonstrated through a random-slope model and a logistic ordinal regression. Materials and datasets are provided online, allowing anyone with Stata to follow along.
\end{abstract}

Keywords: Structural Equation Modeling · Growth Curve Modeling · Mediation · Software.

\section{Introduction}

Structural equation modeling (SEM) is a multivariate statistical analysis framework that allows simultaneous estimation of a system of equations. SEM can be used to fit a wide range of models, including those involving measurement error and latent constructs. This tutorial will demonstrate how to fit a variety of SEMs using Stata statistical software (StataCorp, 2021). Specifically, we will fit models in Stata with both measurement and structural components, as well as those with random effects and generalized responses. We will assess model fit, compute modification indices, estimate mediation effects, conduct group analysis, and more. First, however, we will begin with an introduction to Stata itself. Familiarity with SEM theory and concepts is assumed.

Stata is a complete, integrated software package that provides tools for data manipulation, visualization, statistics, and automated reporting. The Data Editor, Variables window, and Properties window can be used to view and edit your dataset and to manage variables, including their names, labels, value labels, notes, formats, and storage types. Commands can be typed into the Command window, or generated through the point-and-click interface. Log files keep a record of every command issued in a session, while do-files save selected commands to allow users to replicate their work. To learn more about a command, 
you can type help followed by the command name in the Command window and the Viewer window will open with the help file and provide links to further documentation. Stata's documentation consists of over 17,000 pages detailing each feature in Stata including the methods and formulas and fully worked examples.
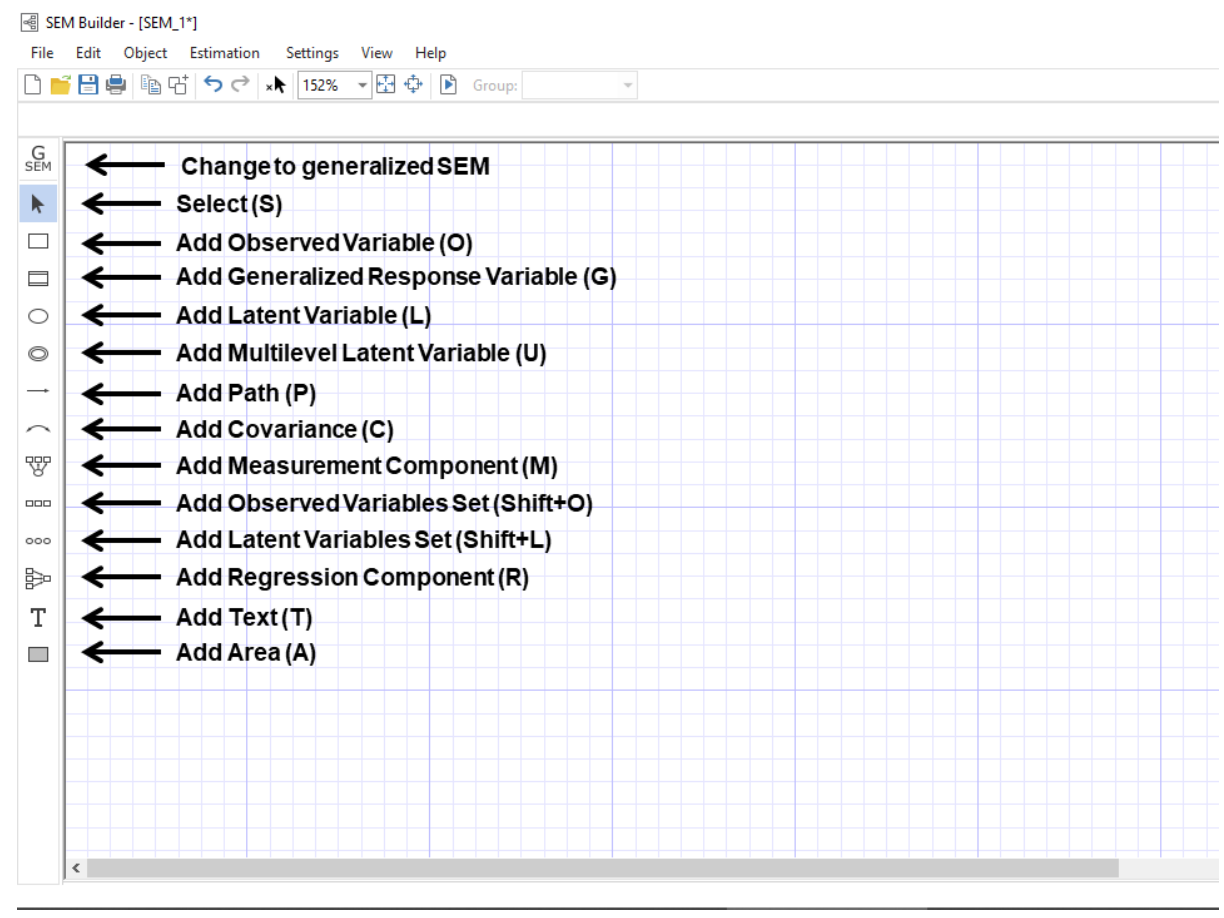

Figure 1. SEM Builder

There are three ways to fit SEMs in Stata: the sem command, the gsem command, and through the SEM Builder. The sem command is for fitting standard linear SEMs. It is quicker and has more features for testing and interpreting results than gsem. The gsem command is for fitting models with generalized responses, such as binary, count, or categorical responses, models with random effects, and mixture models. Both sem and gsem models can be fit via path diagrams using the SEM Builder. You can open the SEM Builder window by typing sembuilder into the Command window. See the interface in Figure 1; click the tools you need on the left, or type their shortcuts shown in the parentheses. To fit gsem models, the GSEM button must first be selected. Estimation and diagram settings can be changed using the menus at the top. The Estimate button fits the model. Path diagrams can be saved as .stsem files to be modified later, or can be exported to a variety of image formats (for example see Figure 2). Although this tutorial will focus on the sem and gsem commands, the Builder shares the same 
functionality. You can watch a demonstration with the SEM Builder on the StataCorp YouTube Channel: https : //www - youtube. com/watch?v=HeQcha3C8Fk

To download the datasets, do-file, and path diagrams, you can type the following into Stata's Command window:

- net from http://www.stata.com/users/mcain/JBDS_SEM

Clicking on the SEMtutorial link will download the materials to your current working directory. To open the do-file with the commands we'll be using, you can type

- doedit SEMtutorial

Commands can either be executed from the do-file or typed into the Command window. We'll start by loading and exploring our first dataset. These data contain observations on four indicators for socioeconomic status of high school students as well as their math scores, school types (private or public), and the student-teacher ratio of their school. Alternatively, we could have used a summary statistics dataset containing means, variances, and correlations of the variables rather than observations.

\begin{tabular}{|c|c|c|c|c|c|c|}
\hline Variable & Obs & Unique & Mean & Min & $\operatorname{Max}$ & Label \\
\hline schtype & 519 & 2 & .61079 & 0 & 1 & School type \\
\hline ratio & 519 & 14 & 16.75723 & 10 & 28 & Student-Teacher ratio \\
\hline math & 519 & 42 & 51.72254 & 30 & 71 & Math score \\
\hline ses 1 & 519 & 5 & 1.982659 & 0 & 4 & SES item 1 \\
\hline ses2 & 519 & 5 & 2.003854 & 0 & 4 & SES item 2 \\
\hline ses3 & 519 & 5 & 2.003854 & 0 & 4 & SES item 3 \\
\hline ses 4 & 519 & 5 & 2.003854 & 0 & 4 & SES item 4 \\
\hline
\end{tabular}

\section{Fitting models with the sem command}

\subsection{Path Analysis}

Let's start our analysis by fitting the one-factor confirmatory factor analysis (CFA) model shown in Figure 2. Using the sem command, paths are specified in parentheses and the direction of the relationships are specified using arrows, i.e. $(\mathrm{x}->\mathrm{y})$. Arrows can point in either direction, $(\mathrm{x}->\mathrm{y})$ or $(\mathrm{y}<-\mathrm{x})$. Paths can be specified individually, or multiple paths can be specified within a single set of parentheses, ( $x 1$ x2 x3 $->y$ ). By default, Stata assumes that all lower-case variables are observed and uppercase variables are latent. You can change these settings using the nocapslatent and the latent() options. In Stata, options are always added after a comma. We'll see plenty of examples of this later. 


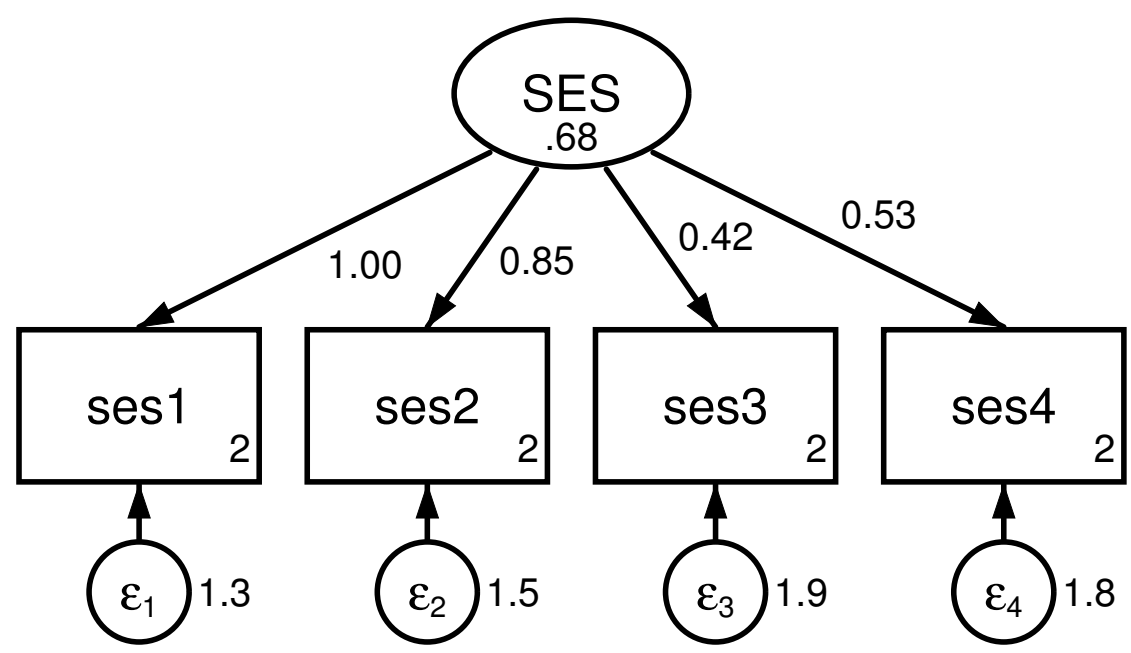

Figure 2. One-factor CFA measuring socioeconomic status (SES)

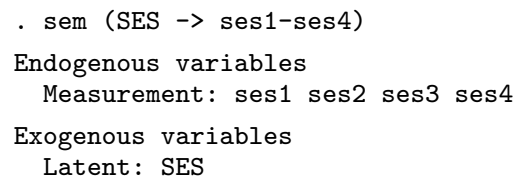

\begin{tabular}{|c|c|c|c|c|c|c|}
\hline & Coefficient & $\begin{array}{l}\text { OIM } \\
\text { std. err. }\end{array}$ & $z$ & $P>|z|$ & {$[95 \%$ conf. } & interval] \\
\hline \multicolumn{7}{|l|}{$\begin{array}{l}\text { Measurement } \\
\text { ses1 }\end{array}$} \\
\hline SES & 1 & (constrained) & & & & \\
\hline _cons & 1.982659 & .0620424 & 31.96 & 0.000 & 1.861058 & 2.10426 \\
\hline \multicolumn{7}{|l|}{ ses2 } \\
\hline SES & .8481035 & .1962358 & 4.32 & 0.000 & .4634884 & 1.232719 \\
\hline _cons & 2.003854 & .0620169 & 32.31 & 0.000 & 1.882303 & 2.125404 \\
\hline \multicolumn{7}{|l|}{ ses 3} \\
\hline SES & .416385 & .1331306 & 3.13 & 0.002 & .1554539 & .6773161 \\
\hline _cons & 2.003854 & .062017 & 32.31 & 0.000 & 1.882302 & 2.125405 \\
\hline
\end{tabular}




\begin{tabular}{r|rrrrrr} 
SES & .5315065 & .1517342 & 3.50 & 0.000 & .234113 & .8289001 \\
_cons & 2.003854 & .062017 & 32.31 & 0.000 & 1.882302 & 2.125405 \\
\hline $\operatorname{var}(\mathrm{e} . \mathrm{ses} 1)$ & 1.317579 & .1855509 & & & .9997798 & 1.736397 \\
$\operatorname{var}(\mathrm{e} . \mathrm{ses} 2)$ & 1.506881 & .1493285 & & & 1.240872 & 1.829916 \\
$\operatorname{var}(\mathrm{e} . \mathrm{ses} 3)$ & 1.878203 & .1257611 & & & 1.647204 & 2.141595 \\
$\operatorname{var}(\mathrm{e} . \mathrm{ses} 4)$ & 1.803979 & .1287389 & & & 1.568507 & 2.074801 \\
$\operatorname{var}(\mathrm{SES})$ & .6801844 & .1908617 & & & .3924434 & 1.178898 \\
\hline
\end{tabular}

LR test of model vs. saturated: $\operatorname{chi2}(2)=11.03$

Prob $>$ chi2 $=0.0040$

Viewing the results, we see that by default Stata constrained the first factor loading to be 1 and estimated the variance of the latent variable. If, instead, we would like to constrain the variance and estimate all four factor loadings, we could use the var () option. Constraints in any part of the model can be specified using the @ symbol. To save room, syntax and results for this and the remaining models will be shown on their path diagrams; see Figure 3.

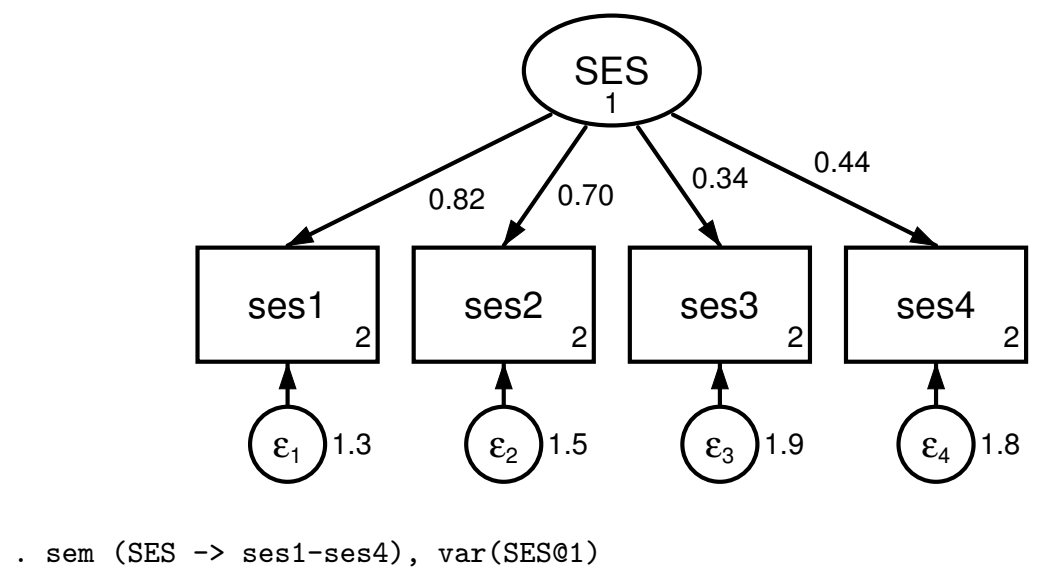

Figure 3. One-factor CFA with constrained variance.

Specifying structural paths is no different from specifying measurement paths. We can add math score to our model and hypothesize that socioeconomic status influences expected math performance. This model is shown in Figure 4; we've added the standardized option to get standardized coefficients. With every increase of one standard deviation in SES, math score is expected to increase by 0.45 standard deviations. 


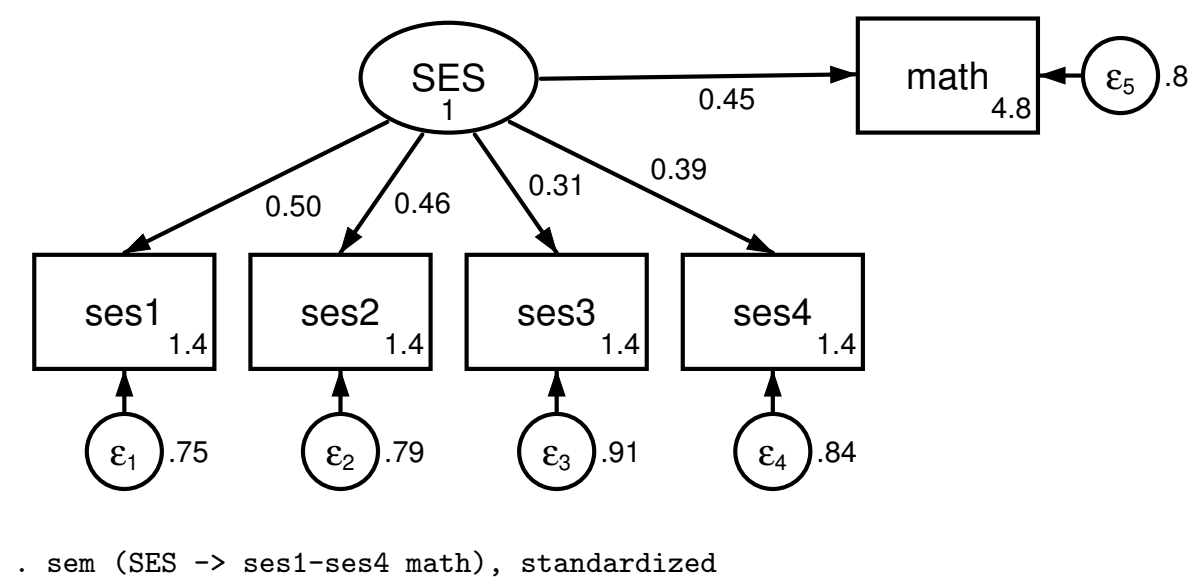

Figure 4. SES influences math scores.

To get fit indices for our model, we can use the postestimation command estat gof after any sem model. Add the stats (all) option to see all fit indices.

\begin{tabular}{|c|c|c|}
\hline Fit statistic & Value & Description \\
\hline $\begin{array}{r}\text { Likelihood ratio } \\
\text { chi2_ms }(5) \\
\text { p > chi2 } \\
\text { chi2_bs }(10) \\
\text { p }>\text { chi2 }\end{array}$ & $\begin{array}{r}17.689 \\
0.003 \\
150.126 \\
0.000\end{array}$ & $\begin{array}{l}\text { model vs. saturated } \\
\text { baseline vs. saturated }\end{array}$ \\
\hline 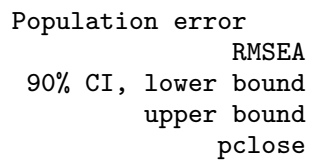 & $\begin{array}{l}0.070 \\
0.037 \\
0.107 \\
0.147\end{array}$ & $\begin{array}{l}\text { Root mean squared error of approximation } \\
\text { Probability RMSEA }<=0.05\end{array}$ \\
\hline $\begin{array}{r}\text { Information criteria } \\
\text { AIC } \\
\text { BIC }\end{array}$ & $\begin{array}{l}11157.441 \\
11221.219\end{array}$ & $\begin{array}{l}\text { Akaike's information criterion } \\
\text { Bayesian information criterion }\end{array}$ \\
\hline $\begin{array}{r}\text { Baseline comparison } \\
\text { CFI } \\
\text { TLI }\end{array}$ & $\begin{array}{l}0.909 \\
0.819\end{array}$ & $\begin{array}{l}\text { Comparative fit index } \\
\text { Tucker-Lewis index }\end{array}$ \\
\hline $\begin{array}{r}\text { Size of residuals } \\
\text { SRMR } \\
\text { CD }\end{array}$ & $\begin{array}{l}0.040 \\
0.532\end{array}$ & $\begin{array}{l}\text { Standardized root mean squared residual } \\
\text { Coefficient of determination }\end{array}$ \\
\hline
\end{tabular}

Satorra-Bentler adjusted model fit indices can be obtained by adding the vce (sbentler) option to our model statement and recalculating the model fit 
indices. This option still uses maximum likelihood estimation, the default, but adjusts the standard errors and the fit indices. Alternatively, estimation can be changed to asymptotic distribution-free or full-information maximum likelihood for missing values using the method (adf) or method (mlmv) options, respectively. For this example, we'll use the Satorra-Bentler adjustment (Satorra \& Bentler, 1994). First, we'll store the current model to use again later.

\begin{tabular}{|c|c|c|c|c|c|c|}
\hline \multicolumn{7}{|c|}{$\begin{array}{l}\text { Endogenous variables } \\
\quad \text { Measurement: ses } 1 \text { ses } 2 \text { ses } 3 \text { ses } 4 \text { math }\end{array}$} \\
\hline \multicolumn{7}{|c|}{$\begin{array}{l}\text { Exogenous variables } \\
\text { Latent: SES }\end{array}$} \\
\hline \multicolumn{7}{|c|}{$\begin{array}{lll}\text { Iteration } 0: & \log \text { pseudolikelihood }=-5564.2324 \\
\text { Iteration 1: } & \text { log pseudolikelihood }=-5563.7459 \\
\text { Iteration 2: } & \text { log pseudolikelihood }=-5563.7204 \\
\text { Iteration 3: } & \text { log pseudolikelihood }=-5563.7204\end{array}$} \\
\hline \multicolumn{5}{|c|}{$\begin{array}{l}\text { Structural equation model } \\
\text { Estimation method: ml }\end{array}$} & Number of & $\mathrm{obs}=519$ \\
\hline \multicolumn{7}{|c|}{ Log pseudolikelihood $=-5563.7204$} \\
\hline & $\begin{array}{r}S \\
\text { Coefficient }\end{array}$ & $\begin{array}{l}\text { torra-Bent } \\
\text { std. err. }\end{array}$ & $\mathrm{z}$ & $P>|z|$ & {$[95 \%$ conf } & interval] \\
\hline \multicolumn{7}{|l|}{$\begin{array}{l}\text { Measurement } \\
\quad \text { ses } 1\end{array}$} \\
\hline $\begin{array}{r}\text { SES } \\
\text { _cons }\end{array}$ & $\begin{array}{r}1 \\
1.982659\end{array}$ & $\begin{array}{l}\text { (constrain } \\
.0621024\end{array}$ & d) 31.93 & 0.000 & 1.860941 & 2.104377 \\
\hline \multicolumn{7}{|l|}{$\operatorname{ses} 2$} \\
\hline $\begin{array}{r}\text { SES } \\
\text { cons }\end{array}$ & $\begin{array}{l}.9278593 \\
2.003854\end{array}$ & $\begin{array}{r}.169484 \\
.0620769\end{array}$ & $\begin{array}{r}5.47 \\
32.28\end{array}$ & $\begin{array}{l}0.000 \\
0.000\end{array}$ & $\begin{array}{l}.5956767 \\
1.882185\end{array}$ & $\begin{array}{l}1.260042 \\
2.125522\end{array}$ \\
\hline \multicolumn{7}{|l|}{ ses3 } \\
\hline $\begin{array}{r}\text { SES } \\
\text { _cons }\end{array}$ & $\begin{array}{r}.620192 \\
2.003854\end{array}$ & $\begin{array}{l}.1438296 \\
.0620769\end{array}$ & $\begin{array}{r}4.31 \\
32.28\end{array}$ & $\begin{array}{l}0.000 \\
0.000\end{array}$ & $\begin{array}{l}.3382912 \\
1.882185\end{array}$ & $\begin{array}{l}.9020928 \\
2.125522\end{array}$ \\
\hline \multicolumn{7}{|l|}{$\operatorname{ses} 4$} \\
\hline $\begin{array}{r}\text { SES } \\
\text { _cons }\end{array}$ & $\begin{array}{l}.7954927 \\
2.003854\end{array}$ & $\begin{array}{l}.1580751 \\
.0620769\end{array}$ & $\begin{array}{r}5.03 \\
32.28\end{array}$ & $\begin{array}{l}0.000 \\
0.000\end{array}$ & $\begin{array}{l}.4856712 \\
1.882185\end{array}$ & $\begin{array}{l}1.105314 \\
2.125522\end{array}$ \\
\hline \multicolumn{7}{|l|}{ math } \\
\hline $\begin{array}{r}\text { SES } \\
\text { _cons }\end{array}$ & $\begin{array}{l}6.858402 \\
51.72254\end{array}$ & $\begin{array}{l}1.335695 \\
.4700825\end{array}$ & $\begin{array}{r}5.13 \\
110.03\end{array}$ & $\begin{array}{l}0.000 \\
0.000\end{array}$ & $\begin{array}{r}4.240488 \\
50.8012\end{array}$ & $\begin{array}{l}9.476315 \\
52.64389\end{array}$ \\
\hline $\operatorname{var}(e \cdot \operatorname{ses} 1)$ & 1.506551 & .1203549 & & & 1.2882 & 1.761913 \\
\hline $\operatorname{var}(e \cdot \operatorname{ses} 2)$ & 1.573228 & .1228219 & & & 1.350014 & 1.833348 \\
\hline $\operatorname{var}(e \cdot \operatorname{ses} 3)$ & 1.807189 & .0933725 & & & 1.633143 & 1.999783 \\
\hline $\operatorname{var}(\mathrm{e} \cdot \operatorname{ses} 4)$ & 1.685282 & .1047979 & & & 1.491906 & 1.903724 \\
\hline $\operatorname{var}$ (e.math) & 91.36045 & 6.594622 & & & 79.3079 & 105.2447 \\
\hline $\operatorname{var}(\mathrm{SES})$ & .4912213 & .1193158 & & & .3051572 & .7907347 \\
\hline $\begin{array}{l}\text { LR test of mod } \\
\text { Satorra-Bentle }\end{array}$ & $\begin{array}{l}\text { l vs. satur } \\
\text { scaled tes }\end{array}$ & $\begin{array}{ll}\text { ted } & \operatorname{chi} 2( \\
: & \operatorname{chi} 2(\end{array}$ & $\begin{array}{l}=17.6 \\
=17.8\end{array}$ & & $\begin{array}{l}\text { Prob }>\mathrm{ch} \\
\text { Prob }>\mathrm{ch}\end{array}$ & $\begin{array}{l}=0.0034 \\
=0.0032\end{array}$ \\
\hline
\end{tabular}




\begin{tabular}{|c|c|c|}
\hline Fit statistic & Value & Description \\
\hline $\begin{array}{r}\text { Likelihood ratio } \\
\text { chi2_ms (5) } \\
\text { p > chi2 } \\
\text { chi2_bs (10) } \\
\text { p > chi2 } \\
\text { Satorra-Bentler } \\
\text { chi2sb_ms }(5) \\
\text { p }>\text { chi2 } \\
\text { chi2sb_bs }(10) \\
\text { p }>\text { chi2 }\end{array}$ & $\begin{array}{r}17.689 \\
0.003 \\
150.126 \\
0.000\end{array}$ & $\begin{array}{l}\text { model vs. saturated } \\
\text { baseline vs. saturated }\end{array}$ \\
\hline $\begin{array}{l}\text { Population error } \\
\text { RMSEA } \\
90 \% \text { CI, lower bound } \\
\text { upper bound } \\
\text { pclose }\end{array}$ & $\begin{array}{l}0.070 \\
0.037 \\
0.107 \\
0.147\end{array}$ & $\begin{array}{l}\text { Root mean squared error of approximation } \\
\text { Probability RMSEA }<=0.05\end{array}$ \\
\hline $\begin{array}{r}\text { Satorra-Bentler } \\
\text { RMSEA_SB }\end{array}$ & 0.070 & Root mean squared error of approximation \\
\hline $\begin{array}{r}\text { Information criteria } \\
\text { AIC } \\
\text { BIC }\end{array}$ & $\begin{array}{l}11157.441 \\
11221.219\end{array}$ & $\begin{array}{l}\text { Akaike's information criterion } \\
\text { Bayesian information criterion }\end{array}$ \\
\hline $\begin{array}{r}\text { Baseline comparison } \\
\text { CFI } \\
\text { TLI }\end{array}$ & $\begin{array}{l}0.909 \\
0.819\end{array}$ & $\begin{array}{l}\text { Comparative fit index } \\
\text { Tucker-Lewis index }\end{array}$ \\
\hline $\begin{array}{r}\text { Satorra-Bentler } \\
\text { CFI_SB } \\
\text { TLI_SB }\end{array}$ & $\begin{array}{l}0.911 \\
0.821\end{array}$ & $\begin{array}{l}\text { Comparative fit index } \\
\text { Tucker-Lewis index }\end{array}$ \\
\hline $\begin{array}{r}\text { Size of residuals } \\
\text { SRMR } \\
\text { CD }\end{array}$ & $\begin{array}{l}0.040 \\
0.532\end{array}$ & $\begin{array}{l}\text { Standardized root mean squared residual } \\
\text { Coefficient of determination }\end{array}$ \\
\hline
\end{tabular}

The SB-adjusted CFI is still rather low, 0.91, indicating poor fit. We can use estat mindices to compute modification indices that can be used to check for paths and covariances that could be added to the model to improve fit. First, we'll need to restore our original model.

. estimates restore $\mathrm{m} 1$ 
- estat mindices

Modification indices

\begin{tabular}{l|rrrrr}
\hline & & & & & Standard \\
& MI & df & P>MI & EPC & $\begin{array}{r}\text { EPC } \\
\text { cov(e.ses1,e.ses2) }\end{array}$ \\
cov(e.ses2,e.ses3) & 16.565 & 1 & 0.00 & .4818524 & .312987 \\
$\operatorname{cov}($ e.ses3,e.ses4) & 4.404 & 1 & 0.02 & -.2203899 & -.1307056 \\
\hline
\end{tabular}

EPC is expected parameter change.

The MI, df, and P>MI are the estimated chi-squared test statistic, degrees of freedom, and $p$ value of the score test testing the statistical significance of the constrained parameter. By default, only parameters that would significantly $(p<0.05)$ improve the model are reported. The EPC is the amount that the parameter is expected to change if the constraint is relaxed. According to these results, we see that there is a stronger relationship between the first and second indicator for SES than would be expected given our model, MI $=16.57, p<0.001$. We could consider adding a residual covariance between these two indicators to our model using the $\operatorname{cov}()$ option. We use the e. prefix to refer to a residual variance of an endogenous variable; see Figure 5.

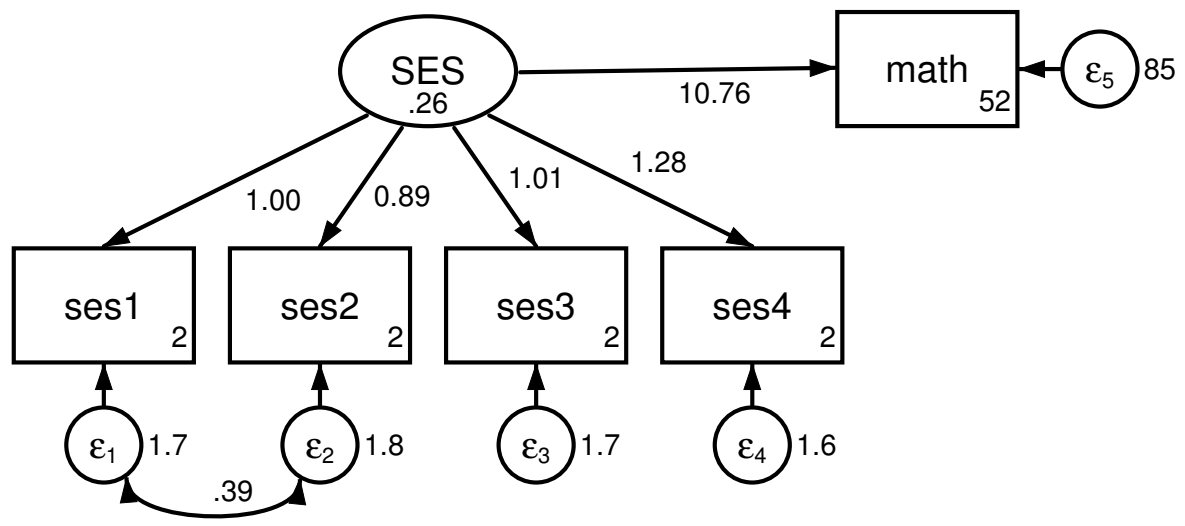

. $\operatorname{sem}(\mathrm{SES} \rightarrow \operatorname{ses} 1-\operatorname{ses} 4$ math), $\operatorname{cov}($ e.ses $1 * e . s e s 2)$

Figure 5. CFA with residual covariance.

One potential explanation of the effect that SES has on math score is that students of higher SES attend schools with smaller student to teacher ratios. We can test this hypothesis using the mediation model shown in Figure 6. Here, we get estimates of the direct effects between each of our variables, but what 
we would really like to test is the indirect effect between SES and math through ratio. We can get direct effects, indirect effects, and total effects of mediation models with the postestimation command estat teffects.

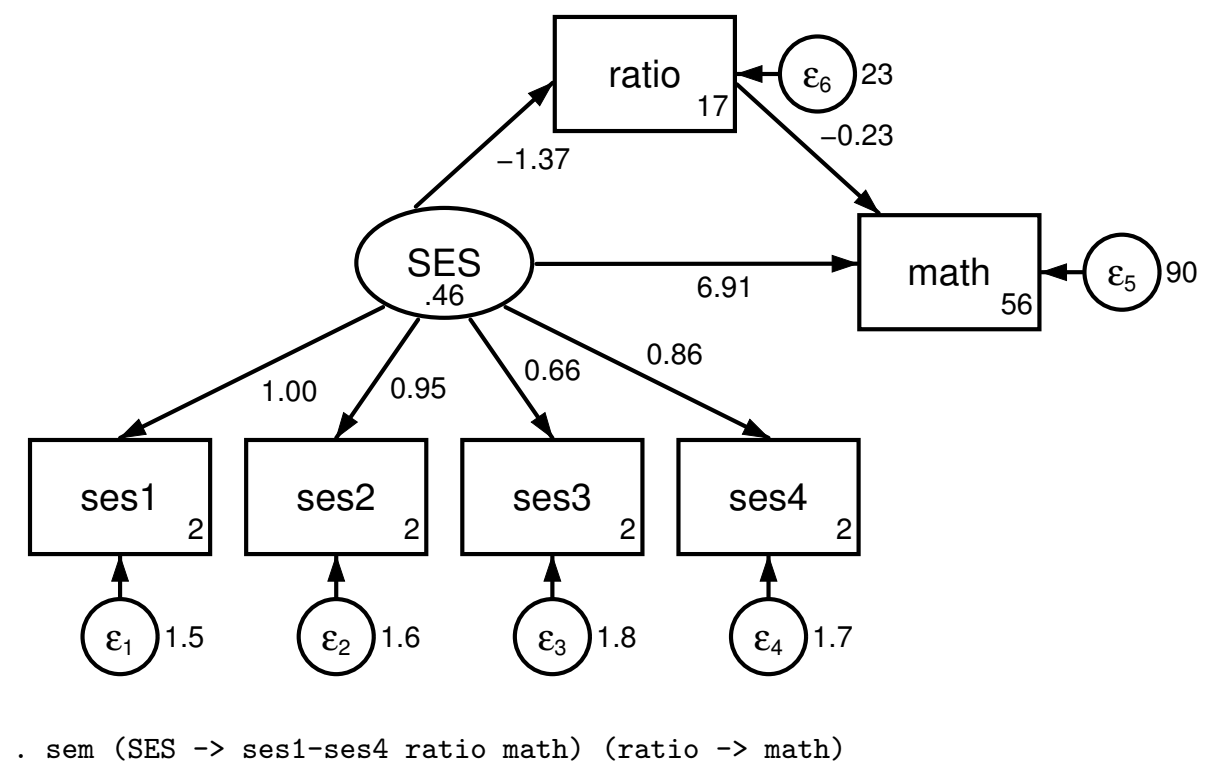

Figure 6. Student-teacher ratio mediates the effect of SES on math score.

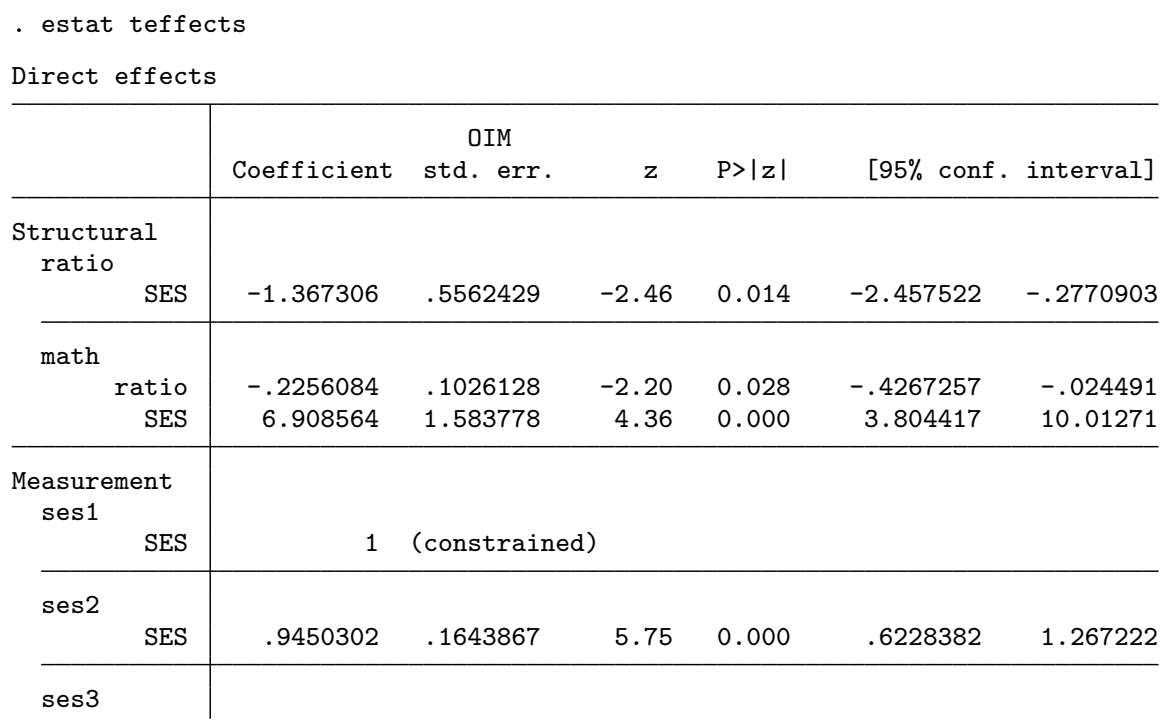




\begin{tabular}{ll|llllll} 
& SES & .6632608 & .1725434 & 3.84 & 0.000 & .3250819 & 1.00144 \\
\hline Ses4 & & & & & & & \\
& SES & .8574695 & .2012317 & 4.26 & 0.000 & .4630625 & 1.251876 \\
\hline
\end{tabular}

Indirect effects

\begin{tabular}{|c|c|c|c|c|c|c|}
\hline & Coefficient & $\begin{array}{c}\text { OIM } \\
\text { std. err. }\end{array}$ & $\mathbf{z}$ & $P>|z|$ & {$[95 \%$ conf. } & interval] \\
\hline $\begin{array}{l}\text { Structural } \\
\text { ratio } \\
\qquad \text { SES }\end{array}$ & 0 & (no path) & & & & \\
\hline $\begin{array}{r}\text { math } \\
\text { ratio } \\
\text { SES }\end{array}$ & $\begin{array}{r}0 \\
.3084758\end{array}$ & $\begin{array}{r}\text { (no path) } \\
.1451257\end{array}$ & 2.13 & 0.034 & .0240346 & .5929169 \\
\hline $\begin{array}{l}\text { Measurement } \\
\text { ses } 1 \\
\\
\text { SES }\end{array}$ & 0 & (no path) & & & & \\
\hline $\begin{array}{l}\text { ses } 2 \quad \text { SES } \\
\end{array}$ & 0 & (no path) & & & & \\
\hline $\begin{array}{l}\text { ses3 } \\
\text { SES }\end{array}$ & 0 & (no path) & & & & \\
\hline $\begin{array}{l}\text { ses4 } \\
\text { SES }\end{array}$ & 0 & (no path) & & & & \\
\hline
\end{tabular}

Total effects

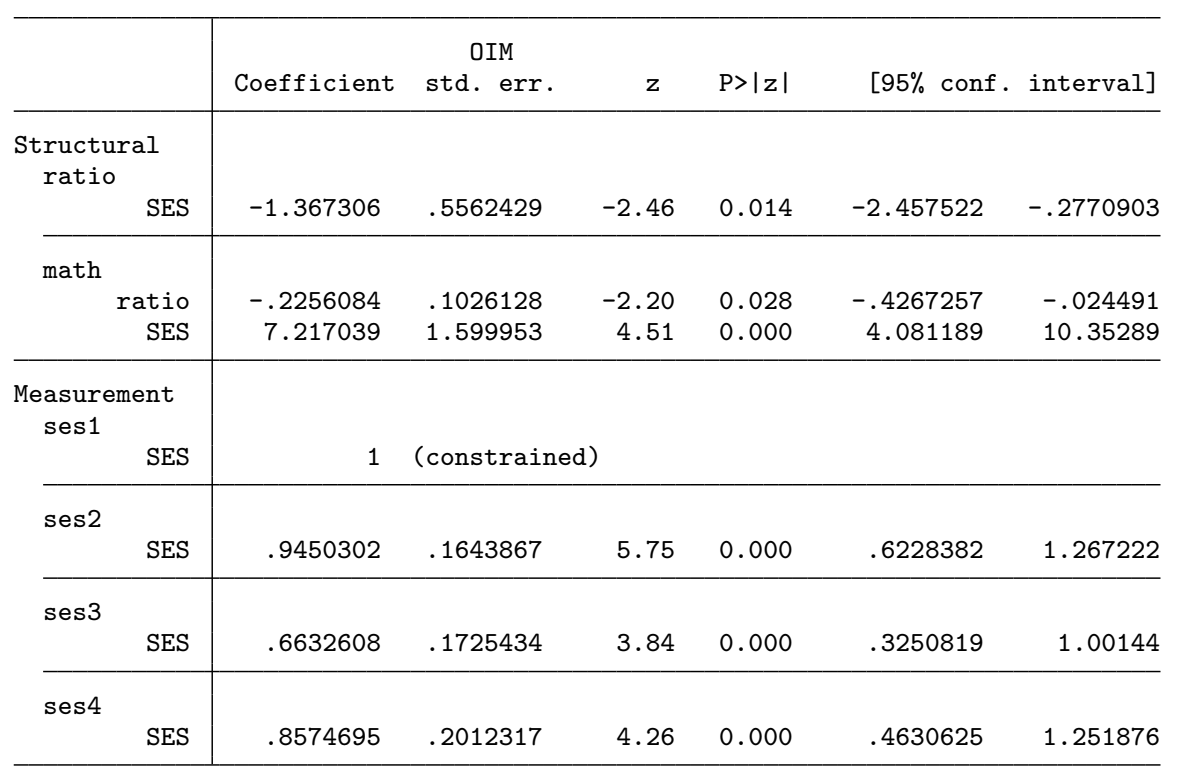


In the second group of the output, we see that the mediation effect is not statistically significant, $z=1.48, p=0.138$. We may consider bootstrapping this effect to get a more powerful test. We can do this with the bootstrap command. First, we need to get labels for the effects we would like to test. We can get these by replaying our model results with the coeflegend option. We can use these labels to construct an expression for the mediation effect that we're calling indirect. We put this expression in parentheses after bootstrap and put any bootstrapping options after a comma; then, we put the model and its options after a colon. Multiple expressions can be included using multiple parentheses sets.

\begin{tabular}{|c|c|c|c|}
\hline \multicolumn{3}{|c|}{$\begin{array}{l}\text { Structural equation model } \\
\text { Estimation method: ml } \\
\text { Log likelihood }=-7117.1959 \\
\text { (1) [ses1] SES }=1\end{array}$} & \multirow{2}{*}{ Number of obs $=519$} \\
\hline & Coefficient & Legend & \\
\hline \multicolumn{3}{|l|}{$\begin{array}{l}\text { Structural } \\
\quad \text { ratio }\end{array}$} & \\
\hline $\begin{array}{r}\text { SES } \\
\text { _cons }\end{array}$ & $\begin{array}{r}-1.367306 \\
16.75723\end{array}$ & $\begin{array}{l}\text { _b[ratio:SES }] \\
\text { _b[ratio:_cons }]\end{array}$ & \\
\hline \multicolumn{3}{|l|}{ math } & \\
\hline $\begin{array}{r}\text { ratio } \\
\text { SES } \\
\text { _cons }\end{array}$ & $\begin{array}{r}-.2256084 \\
6.908564 \\
55.50311\end{array}$ & $\begin{array}{l}\text {-b [math:ratio] } \\
\text {-b[math:SES] } \\
\text { _b [math:_cons] }\end{array}$ & \\
\hline \multicolumn{3}{|l|}{$\begin{array}{l}\text { Measurement } \\
\quad \text { ses1 }\end{array}$} & \\
\hline $\begin{array}{r}\text { SES } \\
\text { _cons }\end{array}$ & $\begin{array}{r}1 \\
1.982659\end{array}$ & $\begin{array}{l}\text { _b[ses } 1: \text { SES }] \\
\text { _b[ses1:_cons }]\end{array}$ & \\
\hline \multicolumn{3}{|l|}{$\operatorname{ses} 2$} & \\
\hline $\begin{array}{r}\text { SES } \\
\text { _cons }\end{array}$ & $\begin{array}{l}.9450302 \\
2.003854\end{array}$ & $\begin{array}{l}\text {-b } \mathrm{b}[\operatorname{ses} 2: \mathrm{SES}] \\
\mathrm{b}\left[\operatorname{ses} 2: \_\operatorname{cons}\right]\end{array}$ & \\
\hline \multicolumn{3}{|l|}{ ses 3} & \\
\hline $\begin{array}{r}\text { SES } \\
\text { _cons }\end{array}$ & $\begin{array}{l}.6632608 \\
2.003854\end{array}$ & $\begin{array}{l}\text {-b[ses3:SES }] \\
\text { _b[ses3:_cons }]\end{array}$ & \\
\hline \multicolumn{3}{|l|}{ ses 4} & \\
\hline $\begin{array}{r}\text { SES } \\
\text {-cons }\end{array}$ & $\begin{array}{l}.8574695 \\
2.003854\end{array}$ & $\begin{array}{l}\text { _b }[\text { ses } 4: \mathrm{SES}] \\
\left.\text { _b[ses } 4: \_ \text {cons }\right]\end{array}$ & \\
\hline $\begin{array}{c}\operatorname{var}(\text { e.ses } 1) \\
\operatorname{var}(\text { e.ses } 2) \\
\operatorname{var}(\text { e.ses } 3) \\
\operatorname{var}(\text { e.ses } 4) \\
\operatorname{var}(\text { e.ratio }) \\
\operatorname{var}(\text { e.math }) \\
\operatorname{var}(\text { SES })\end{array}$ & $\begin{array}{l}1.541523 \\
1.588663 \\
1.795421 \\
1.660672 \\
23.41179 \\
89.51067 \\
.4562495\end{array}$ & 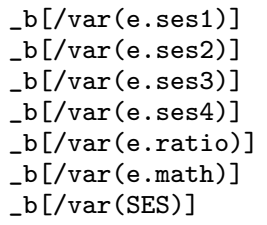 & \\
\hline
\end{tabular}

LR test of model vs. saturated: $\operatorname{chi} 2(8)=21.72$

Prob $>$ chi2 $=0.0055$ 


\begin{tabular}{|c|c|c|c|c|c|}
\hline \multicolumn{5}{|c|}{ Bootstrap results } & $\begin{array}{l}\text { Number of obs }=519 \\
\text { Replications }=1,000\end{array}$ \\
\hline \multirow{2}{*}{$\begin{array}{l}\text { Command } \\
\text { indirect }\end{array}$} & sem (SES -> & ses $1-\operatorname{ses} 4 r$ & io ma & 1) (ratio & $\rightarrow$ math) \\
\hline & $\begin{array}{c}\text { Observed } \\
\text { coefficient }\end{array}$ & $\begin{array}{l}\text { Bootstrap } \\
\text { std. err. }\end{array}$ & $z$ & $P>|z|$ & $\begin{array}{c}\text { Normal-based } \\
\text { [95\% conf. interval] }\end{array}$ \\
\hline indirect & .3084758 & .1932632 & 1.60 & 0.110 & -.070313 \\
\hline
\end{tabular}

We've added the reps (1000) option to compute 1,000 bootstrap replications and the nodots option to suppress displaying a dot for each replication. To get 95 percentile confidence intervals based on our bootstrap sampling distribution, we can follow with the postestimation command estat bootstrap using the percentile option. The resulting confidence interval contains zero so we cannot reject the null hypothesis.

\begin{tabular}{|c|c|c|c|c|c|c|}
\hline \multicolumn{4}{|c|}{ Bootstrap results } & $\begin{array}{l}\text { Number of obs } \\
\text { Replications }\end{array}$ & $\begin{array}{l}= \\
=\end{array}$ & $\begin{array}{r}519 \\
1000\end{array}$ \\
\hline \multicolumn{7}{|c|}{$\begin{array}{l}\text { Command: sem (SES } \rightarrow \text { ses1-ses4 ratio math) (ratio } \rightarrow \text { math) } \\
\text { indirect: _b[ratio:SES]*_b[math:ratio] }\end{array}$} \\
\hline & $\begin{array}{r}\text { Observed } \\
\text { coefficient }\end{array}$ & Bias & $\begin{array}{l}\text { Bootstrap } \\
\text { std. err. }\end{array}$ & {$[95 \% \operatorname{conf}$} & interval] & \\
\hline indirect & .30847577 & -.0307326 & . 19326315 & -.0707015 & .6837121 & (P) \\
\hline
\end{tabular}

Key: P: Percentile

\subsection{Group Analysis}

Finally, we may consider comparing our mediation across groups. Group analysis can be done in Stata by adding the group() option. We would like to compare students in public schools versus private schools so we will specify schtype as our grouping variable. Then, we can use ginvariant() to specify the types of parameters we would like to constrain across groups. All other variables will be estimated separately for each group. The ginvariant() options are listed in Table 1. If we don't specify any ginvariant option, by default Stata will constrain measurement coefficients and measurement intercepts, ginvariant (mcoef mcons). See the model in Figure 7. Now when we run estat teffects, we will get a separate estimated mediation effect for each group. 

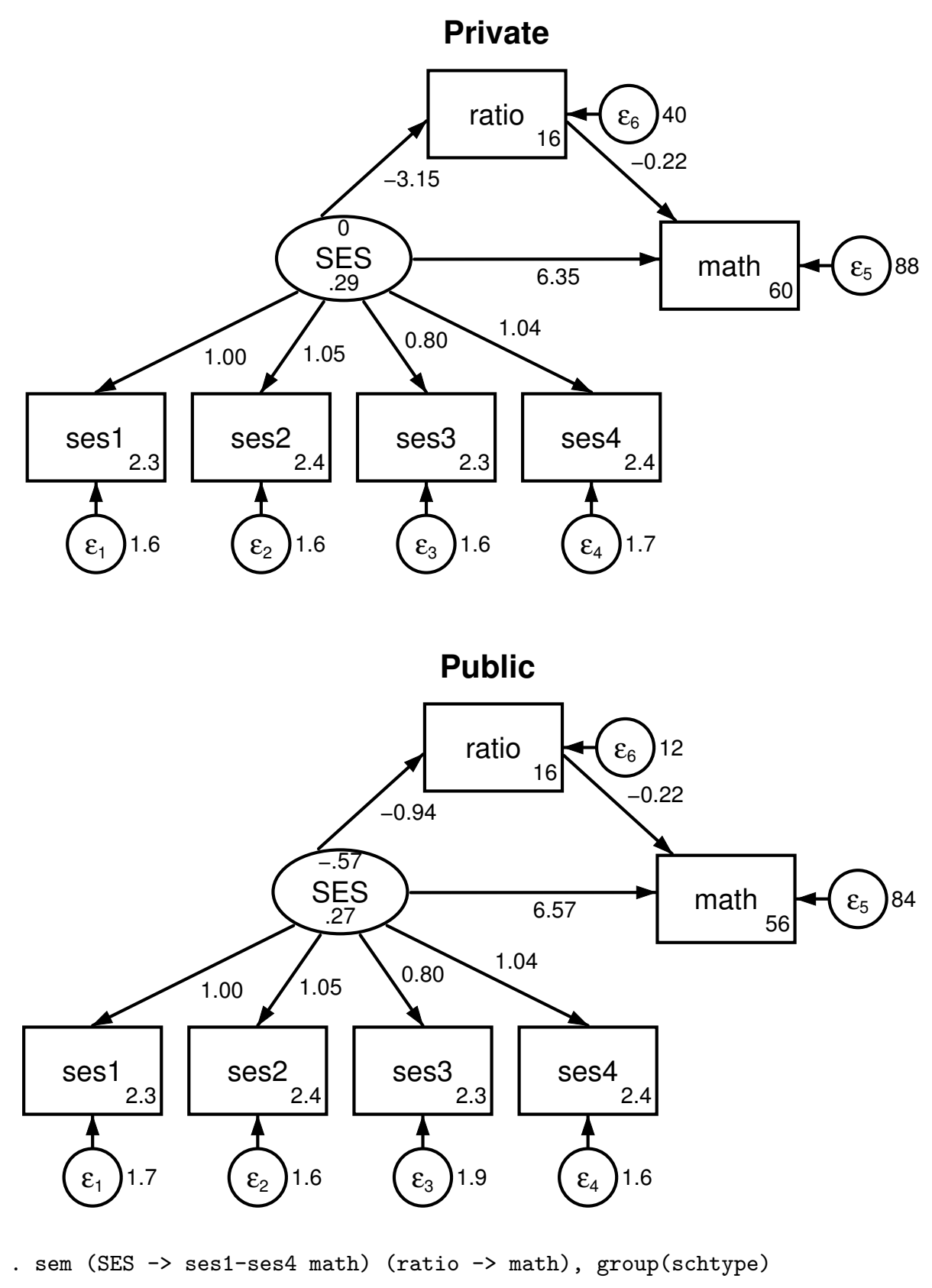

Figure 7. Group analysis. 
- estat teffects, nodirect nototal compact

Indirect effects

\begin{tabular}{|c|c|c|c|c|c|c|}
\hline & Coefficient & $\begin{array}{c}\text { OIM } \\
\text { std. err. }\end{array}$ & $z$ & $P>|z|$ & {$[95 \%$ conf. } & interval] \\
\hline \multirow{2}{*}{\multicolumn{7}{|c|}{$\begin{array}{l}\text { Structural } \\
\text { math } \\
\qquad \text { SES }\end{array}$}} \\
\hline & & & & & & \\
\hline $\begin{array}{r}\text { Private } \\
\text { Public }\end{array}$ & $\begin{array}{l}.7043843 \\
.2035724\end{array}$ & $\begin{array}{l}.4184641 \\
.1710134\end{array}$ & $\begin{array}{l}1.68 \\
1.19\end{array}$ & $\begin{array}{l}0.092 \\
0.234\end{array}$ & $\begin{array}{l}-.1157902 \\
-.1316076\end{array}$ & $\begin{array}{l}1.524559 \\
.5387525\end{array}$ \\
\hline \multicolumn{7}{|l|}{ ratio } \\
\hline \multicolumn{7}{|l|}{$\operatorname{ses} 1$} \\
\hline \multicolumn{7}{|l|}{ ses 2} \\
\hline \multicolumn{7}{|l|}{$\begin{array}{l}\text { Measurement } \\
\text { ses3 }\end{array}$} \\
\hline $\operatorname{ses} 4$ & & & & & & \\
\hline
\end{tabular}

Table 1. ginvariant() suboptions

\begin{tabular}{ll}
\hline Option & Description \\
\hline mcoef & measurement coefficients \\
mcons & measurement intercepts \\
merrvar & covariances of measurement errors \\
scoef & structural coefficients \\
scons & structural intercepts \\
serrvar & covariances of structural errors \\
smerrcov covariances between structural and measurement errors \\
meanex & means of exogenous variables \\
covex & covariances of exogenous variables \\
all & all the above \\
none & none of the above \\
\hline
\end{tabular}

To test whether these mediation effects significantly differ, we can conduct a Wald test with the test or testnl postestimation commands, again using the labels from the coeflegend option. Because mediation effects are nonlinear, we will use testnl. The mediation effects do not significantly differ between groups, $\chi^{2}(1)=1.27, p=0.260$.

- testnl _b[ratio:0bn.schtype\#c.SES]*_b[math:Obn.schtype\#c.ratio]= ///

$>$ _b[ratio:1.schtype\#c.SES]*_b[math:1.schtype\#c.ratio]

(1)_b[ratio:0bn.schtype\#c.SES]*_b[math:0bn.schtype\#c.ratio]

$>$ _b[ratio:1.schtype\#c.SES]*_b[math:1.schtype\#c.ratio]

$$
\operatorname{chi2}(1)=1.27
$$

Prob $>$ chi2 $=\quad 0.2599$ 


\begin{tabular}{|c|c|c|c|c|c|c|}
\hline & \multicolumn{3}{|c|}{ Wald test } & \multicolumn{3}{|c|}{ Score test } \\
\hline & chi2 & $\mathrm{df}$ & $\mathrm{P}>\operatorname{chi} 2$ & chi2 & $d f$ & P>chi2 \\
\hline \multicolumn{7}{|l|}{$\begin{array}{l}\text { Structural } \\
\text { math }\end{array}$} \\
\hline ratio & 0.001 & 1 & 0.9709 & . & . & . \\
\hline SES & 0.005 & 1 & 0.9441 & . & . & . \\
\hline _cons & 1.314 & 1 & 0.2516 & . & . & . \\
\hline \multicolumn{7}{|l|}{ ratio } \\
\hline SES & 1.825 & 1 & 0.1768 & . & . & . \\
\hline _cons & 0.011 & 1 & 0.9147 & . & . & . \\
\hline \multicolumn{7}{|l|}{$\begin{array}{l}\text { Measurement } \\
\text { ses1 }\end{array}$} \\
\hline SES & . & . & . & 1.832 & 1 & 0.1759 \\
\hline _cons & . & . & . & 5.997 & 1 & 0.0143 \\
\hline \multicolumn{7}{|l|}{ ses2 } \\
\hline SES & . & . & . & 0.072 & 1 & 0.7882 \\
\hline _cons & . & . & . & 0.341 & 1 & 0.5592 \\
\hline \multicolumn{7}{|l|}{ ses3 } \\
\hline SES & . & . & . & 0.049 & 1 & 0.8253 \\
\hline _cons & . & . & . & 0.634 & 1 & 0.4259 \\
\hline \multicolumn{7}{|l|}{ ses 4} \\
\hline SES & . & . & . & 1.945 & 1 & 0.1632 \\
\hline _cons & . & . & . & 1.149 & 1 & 0.2838 \\
\hline $\operatorname{var}(\mathrm{e} \cdot \operatorname{ses} 1)$ & 0.189 & 1 & 0.6640 & . & . & . \\
\hline $\operatorname{var}(e \cdot \operatorname{ses} 2)$ & 0.063 & 1 & 0.8023 & . & . & . \\
\hline $\operatorname{var}($ e.ses 3$)$ & 1.011 & 1 & 0.3146 & . & . & . \\
\hline $\operatorname{var}($ e.ses 4$)$ & 0.090 & 1 & 0.7641 & . & . & . \\
\hline $\operatorname{var}$ (e.math) & 0.065 & 1 & 0.7982 & . & . & . \\
\hline $\operatorname{var}$ (e.ratio) & 36.627 & 1 & 0.0000 & . & . & \\
\hline $\operatorname{var}(\mathrm{SES})$ & 0.042 & 1 & 0.8383 & . & . & \\
\hline
\end{tabular}

To test group differences in each direct path, we can use the postestimation command estat ginvariant. These results show us Wald tests evaluating constraining parameters that were allowed to vary across groups and score tests evaluating relaxing constraints. Both are testing whether individual paths significantly differ across groups.

\subsection{Growth Curve Modeling}

The last model we will fit using sem is a growth curve model. This will require a new dataset.

$$
\text { . use crime }
$$




\begin{tabular}{|c|c|c|c|c|}
\hline \multicolumn{5}{|c|}{ Contains data from crime.dta } \\
\hline \multirow{2}{*}{\multicolumn{2}{|c|}{$\begin{array}{l}\text { Observations: } \\
\text { Variables: }\end{array}$}} & 359 & & \\
\hline & & 4 & & $\begin{array}{l}4 \text { Oct } 2012 \text { 16:22 } \\
\text { (_dta has notes) }\end{array}$ \\
\hline Variable & Storage & Display & Value & \\
\hline name & type & format & label & Variable label \\
\hline Incrime0 & float & $\% 9.0 \mathrm{~g}$ & & In(crime rate) in Jan \& Feb \\
\hline lncrime1 & float & $\% 9.0 \mathrm{~g}$ & & In(crime rate) in Mar \& Apr \\
\hline lncrime2 & float & $\% 9.0 \mathrm{~g}$ & & ln(crime rate) in May \& Jun \\
\hline Incrime3 & float & $\% 9.0 \mathrm{~g}$ & & In(crime rate) in Jul \& Aug \\
\hline
\end{tabular}

Sorted by:

These data are from Bollen and Curran (2006); they contain crime rates collected in two-month intervals for the first eight months of 1995 for 359 communities in New York state. We would like to fit a linear growth curve to these data to model how crime rate changed over time. In our model, we can set constraints using the @ symbol as we did before. To constrain all intercepts to 0 , we can add the nocons option. We will also need the means() option. By default, Stata constrains the means of latent variables to 0 . For this model, we would like to estimate them so we need to specify the latent variable names inside means (). We may also consider constraining all the residual variances to equality by constraining each of them to the same arbitrary letter or word, in this case eps. See the model in Figure 8.

The estimated mean log crime rate at the beginning of the study was 5.33 and it increased by an average of 0.14 every two months. We could have fit this same model using gsem. One way we can do this is to simply replace sem with gsem in the command in Figure 8. Alternatively, we can can think of this as a multilevel model, and fit it using gsem's notation for random effects. Let's do that next. 


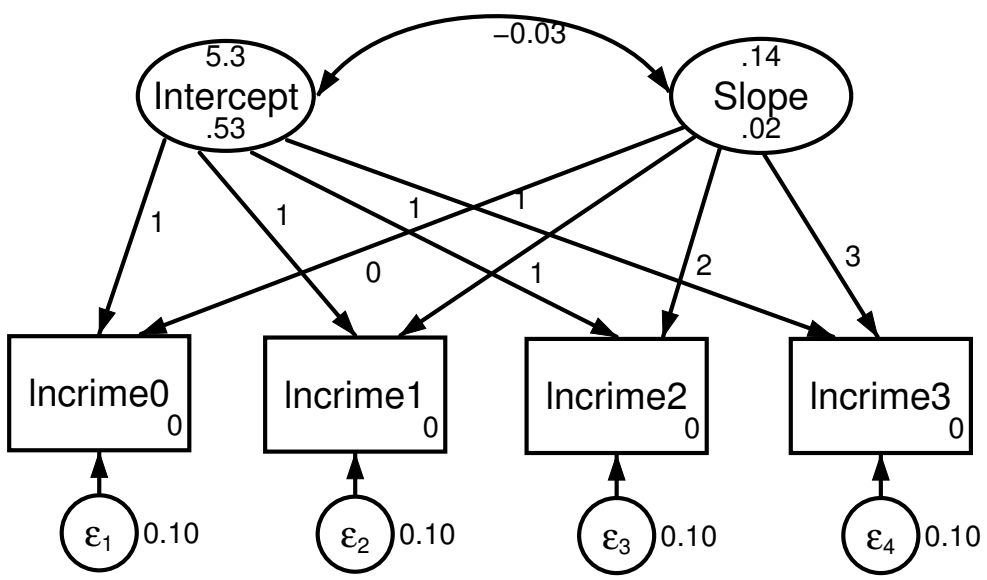

- sem (Intercept@1 -> Incrime0-lncrime3) (Slope -> Incrime0@0 Incrime1@1 lncrime2@2 lncrime3@3), nocons means(Intercept Slope)

$\operatorname{var}($ e.lncrime0-lncrime3@eps)

Figure 8. Growth curve model on crime rate.

\section{$3 \quad$ Fitting models with the gsem command}

\subsection{Models with Random Effects}

The gsem command implements generalizations to the standard linear structural equation model implemented in sem, such as models with generalized-linear response variables, random effects, and categorical latent variables (latent classes). Its syntax is the same as sem, with some different options and postestimation commands. We will start by fitting a random-slope model to the crimes dataset, reproducing the results we obtained with the growth curve model using sem. First, we need to create an observation identification variable and reshape the data into long format.

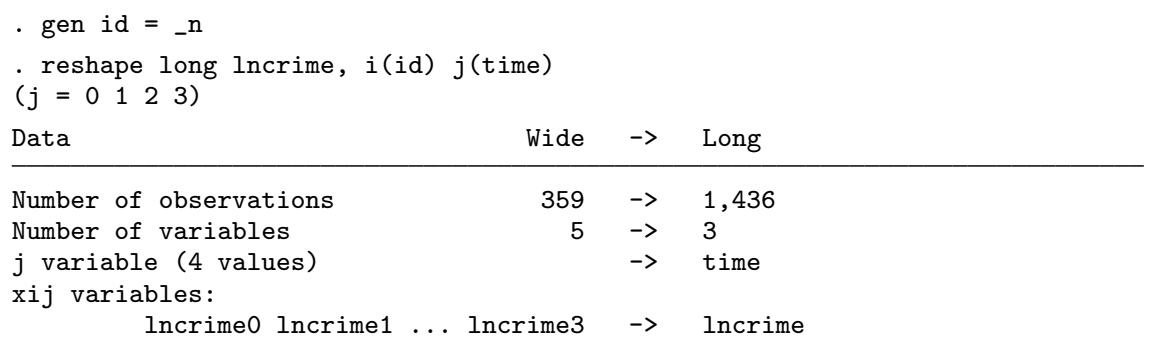




\begin{tabular}{r|rrrrr}
$\begin{array}{c}\text { summarize } \\
\text { Variable }\end{array}$ & Obs & Mean & Std. dev. & Min & Max \\
\hline id & 1,436 & 180 & 103.6701 & 1 & 359 \\
time & 1,436 & 1.5 & 1.118423 & 0 & 3 \\
lncrime & 1,436 & 5.551958 & .7856259 & 2.415164 & 9.575166
\end{tabular}

We now have long-format data in which we have several rows of observations for each individual; we're ready to fit our random-slope model. We specify random effects in gsem by adding brackets enclosing the clustering variable to the latent variable, i.e. Intercept [id]. This tells Stata to include a latent variable in the model called Intercept that has variability at the id level. As with other latent variables, it will have a mean of 0 and an initial factor loading of 1 , so the only parameter this term introduces is a level- 2 variance. Random coefficients can be added to any term by interacting a latent random effect with that variable, i.e. c.time\#Slope [id].

Interactions in Stata are specified using \#; interaction terms are assumed to be factor variables unless prefixed by c. to indicate that they are continuous variables. Contrarily, main-effect terms are assumed to be continuous unless prefixed by i. to indicate that they are factor variables. We'll see this in the next example. This factor variable notation is not available using sem.

See the syntax and results of the random slope model in Figure 9; these results replicate those by sem. In the SEM Builder, random effects are represented as double-bordered ovals labeled with the clustering variable to indicate that they represent variability at the cluster level.

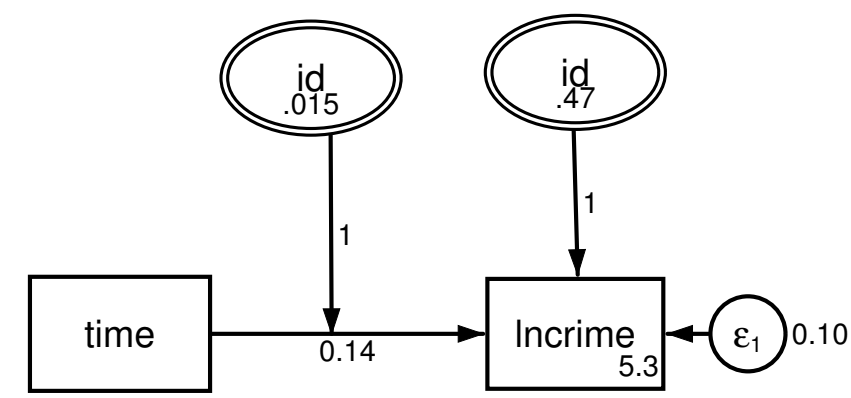

- gsem (Intercept [id] time c.time\#Slope[id] $\rightarrow$ Incrime)

Figure 9. Random-slope model on crime rate. 


\subsection{Models with Generalized Responses}

The gsem command can also be used to fit generalized linear SEMs; that is, SEMs in which an endogenous variable is distributed according to some distribution family and is related to the linear prediction of the model through a link function. See Table 2 for a list of available distribution families and links. Either the family and link can be specified, i.e. family (bernoulli) link(logit), or some combinations have shortcuts that you can specify instead, i.e. logit. For this example, we will return to the first dataset.

Table 2. gsem distribution families and link functions

\begin{tabular}{|c|c|c|c|c|}
\hline \multirow[t]{2}{*}{ family() options } & \multicolumn{4}{|c|}{ link() options } \\
\hline & identity & $\log$ & logit probit & cloglog \\
\hline gaussian & $\mathrm{X}$ & $\mathrm{X}$ & & \\
\hline bernoulli & & & logit probit & cloglog \\
\hline beta & & & $\mathrm{X} \quad \mathrm{X}$ & $\mathrm{X}$ \\
\hline binomial & & & X $\quad X$ & $\mathrm{X}$ \\
\hline ordinal & & & ologit oprobit & ocloglog \\
\hline multinomial & & & mlogit & \\
\hline Poisson & & poisson & & \\
\hline negative binomial & & nbreg & & \\
\hline exponential & & exponential & & \\
\hline Weibull & & weibull & & \\
\hline gamma & & gamma & & \\
\hline loglogistic & & loglogistic & & \\
\hline lognormal & & lognormal & & \\
\hline
\end{tabular}

\begin{tabular}{|c|c|c|c|c|c|c|}
\hline Variable & Obs & Unique & Mean & Min & Max & Label \\
\hline schtype & 519 & 2 & .61079 & 0 & 1 & School type \\
\hline ratio & 519 & 14 & 16.75723 & 10 & 28 & Student-Teacher ratio \\
\hline math & 519 & 42 & 51.72254 & 30 & 71 & Math score \\
\hline ses 1 & 519 & 5 & 1.982659 & 0 & 4 & SES item 1 \\
\hline ses 2 & 519 & 5 & 2.003854 & 0 & 4 & SES item 2 \\
\hline ses3 & 519 & 5 & 2.003854 & 0 & 4 & SES item 3 \\
\hline ses 4 & 519 & 5 & 2.003854 & 0 & 4 & SES item 4 \\
\hline
\end{tabular}

In our previous analysis, we had treated each socioeconomic status Likert item as continuous. Now, we will treat them as ordinal using gsem. Adding the ologit option will fit the measurement model using the ordinal family with a logistic link. We will also use factor variable notation to include indicator 
variables for school type in our analysis. See figure Figure 10. By adding schtype as a factor variable, a dummy variable for each level of schtype is included in the model. The path coefficient for the base level, by default the lowest, is constrained to zero. To get exponentiated coefficients, we can follow with the postestimation command estat eform.

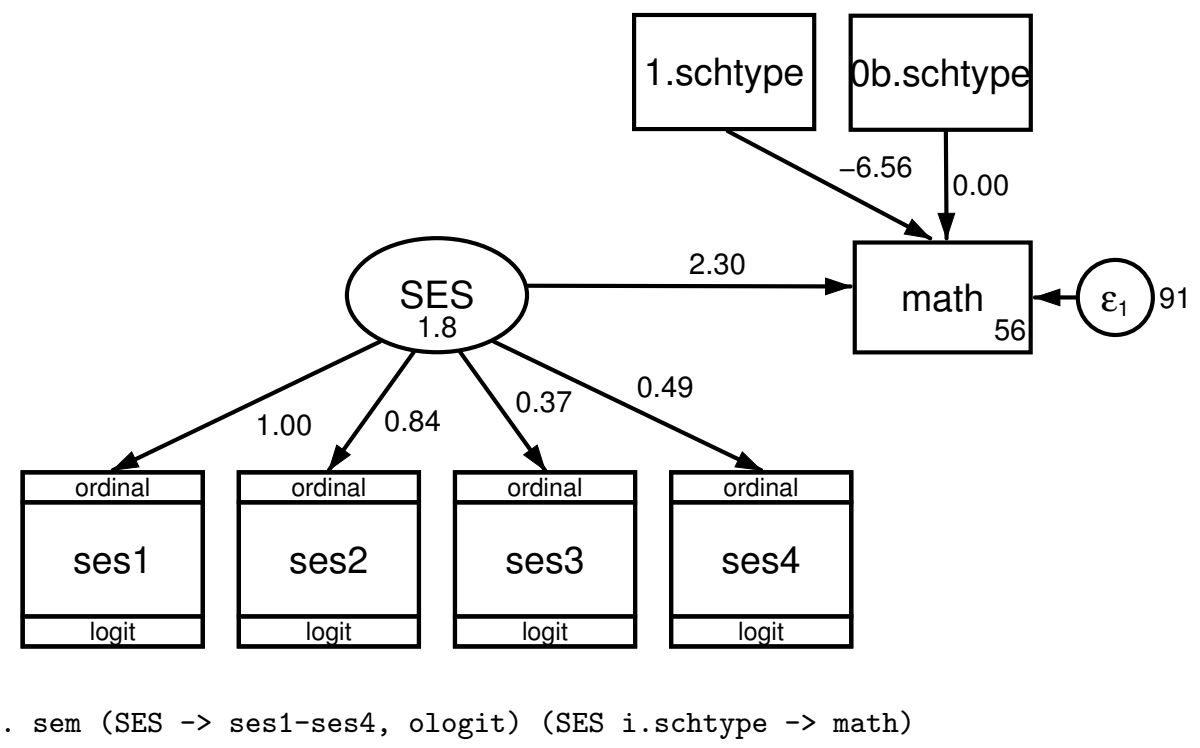

Figure 10. Ordinal logistic regression model.

\begin{tabular}{|c|c|c|c|c|c|c|c|}
\hline & & $\exp (b)$ & Std. err. & $z$ & $\mathrm{P}>|z|$ & {$[95 \%$ conf. } & interval] \\
\hline \multicolumn{8}{|l|}{ ses 1} \\
\hline & SES & 2.718282 & (constrained) & & & & \\
\hline \multicolumn{8}{|l|}{$\operatorname{ses} 2$} \\
\hline & SES & 2.311549 & .483485 & 4.01 & 0.000 & 1.534141 & 3.482899 \\
\hline \multicolumn{8}{|l|}{ ses3 } \\
\hline & SES & 1.449492 & .180061 & 2.99 & 0.003 & 1.136257 & 1.849077 \\
\hline \multicolumn{8}{|l|}{ ses 4} \\
\hline & SES & 1.628133 & .2474222 & 3.21 & 0.001 & 1.208748 & 2.193029 \\
\hline
\end{tabular}




\section{Conclusion}

In this tutorial, we've shown the basics of fitting SEMs in Stata using the sem and gsem commands, and have provided example datasets and syntax online to follow along. We demonstrated confirmatory factor analysis, mediation, group analysis, growth curve modeling, and models with random effects and generalized responses. However, there are many possibilities and options not included in this tutorial, such as latent class analysis models, nonrecursive models, reliability models, mediation models with generalized responses, multivariate randomeffects models, and much more. Visit Stata's documentation to see all the available options for these commands, their methods and formulas, and many more examples online at https://www.stata.com/manuals/sem.pdf.

\section{References}

Bollen, K. A., \& Curran, P. J. (2006). Latent curve models: A structural equation perspective (Vol. 467). John Wiley \& Sons.

Satorra, A., \& Bentler, P. M. (1994). Corrections to test statistics and standard errors in covariance structure analysis. In Latent variables analysis: Applications for developmental research. (pp. 399-419). Sage Publications, Inc.

StataCorp. (2021). Stata statistical software: Release 1\%. StataCorp LLC. 